\title{
Potential mechanisms behind contrast medium-induced nephropathy
}

P B Persson
D Med, D Med Habil
A Patzak
D Med, D Med Habil
Institute of Physiology
Humboldt University
Berlin, Germany

\section{Abstract}

How contrast medium-induced nephropathy (CIN) comes about is poorly understood, although CIN is a common cause of acute renal failure. Hitherto, the various studies performed have led to different interpretations and partially contradictory conclusions. This article aimed to review the mechanisms underlying CIN and to outline existing data obtained with the newer iodinated agents in patients with pre-existing renal failure. Osmolality, which has received considerable attention, is but one of several physico-chemical properties of contrast media (CM). The more recently developed iso-osmolar $\mathrm{CM}$ are dimers, not monomers as the widely used non-ionic low osmolar CM. Thus, in spite of them being iso-osmolar, they have physicochemical features different from other $\mathrm{CM}$, e.g. in terms of viscosity (> 5 fold greater than plasma viscosity), which may be of considerable pathophysiologic and clinical importance. Many experimental studies provide evidence for greater perturbation in renal function with iso-osmolar CM compared with non-ionic lowosmolar CM. Conversely, some clinical trials indicate an advantage of the iso-osmolar CM, although others do not. In this review, the possible causes of CIN are highlighted, including altered rheological properties, perturbation of renal haemodynamics, regional hypoxia, auto- and paracrine factors (adenosine, endothelin, reactive oxygen species) and direct cytotoxic effects. It is concluded that caution must be taken to avoid a false sense of security with the use of iso-osmolar CM.

\section{Introduction}

This review critically surveys recent clinical studies with regard to contrast medium-induced nephropathy (CIN) and focuses on mechanisms believed to mediate CIN, which implies impairment of renal function occurring within 3 days of the intravascular administration of contrast medium (CM) and the absence of an alternative aetiology. ${ }^{1,2}$ An increase in serum creatinine by more than $25 \%$ or $44 \mu \mathrm{mol} / 1$ ( $0.5 \mathrm{mg} / 100 \mathrm{ml}$, within $48-72$ hours of contrast administration) is often taken as a marker for the occurrence of CIN. ${ }^{3-6}$ The serum creatinine concentration typically peaks on the second or third day after exposure to $\mathrm{CM}$ and usually returns to the baseline value within 2 weeks. ${ }^{7,8}$

Generally, CIN is reversible. Nevertheless, the use of CM increases inhospital morbidity, mortality and costs, in particular in those rare cases where dialysis is required. Thus, despite the small relative risk of developing adverse effects, CIN is the third leading cause of acute renal failure in patients who have been admitted, accounting for $10 \%$ of all cases. ${ }^{9,10}$ The incidence of nephropathy induced by lowosmolar CM is low in the general population and has been calculated to be less than $2 \%$. ${ }^{11,12}$ In selected subgroups of patients, however, like those with pre-existing renal insufficiency or diabetes mellitus or a combination of both, the incidence is significantly higher, in the range of $12-50 \%{ }^{10,13-17}$ requiring transient dialysis or progress to end-stage renal disease. ${ }^{18}$

\section{Risk factors}

By far the greatest risk of developing CIN is pre-existing renal impairment combined with diabetes, dehydration, or a combination of both. ${ }^{19}$ Remarkably, however,

Table I. Osmolality and viscosity for various CMs. Note differences in iodine concentrations

\begin{tabular}{|c|c|c|c|}
\hline \multirow[b]{2}{*}{ Generic name } & \multicolumn{3}{|c|}{ Physicochemical properties of CM } \\
\hline & $\begin{array}{l}\text { Iodine concentration } \\
(\mathrm{mg} / \mathrm{ml})\end{array}$ & $\begin{array}{l}\text { Viscosity } \\
\left(\mathrm{mPA} \text { 'S at } 37^{\circ} \mathrm{C}\right)\end{array}$ & $\begin{array}{l}\text { Osmolality } \\
\left(\text { at } 37^{\circ} \mathrm{C} \mathrm{mOsm} / \mathrm{kg} \mathrm{H}_{2} \mathrm{O}\right)\end{array}$ \\
\hline Iopamidol & 300 & 5.25 & 636 \\
\hline Iopromid & 300 & 4.6 & 610 \\
\hline Iohexol & 300 & 5.7 & 690 \\
\hline Ioxaglate & 320 & 7.5 & 600 \\
\hline Iomeprol & 300 & 4.5 & 521 \\
\hline Iodixanol & 320 & 11.4 & 290 \\
\hline Diatrizoat & 292 & 4.0 & 1500 \\
\hline Iopentol & 300 & 6.5 & 640 \\
\hline Iotrolan & 300 & 8.1 & 320 \\
\hline
\end{tabular}


diabetes mellitus per se without renal insufficiency is not a risk factor. ${ }^{15}$ Additional risk factors are: dosage of $\mathrm{CM}$ and type of $\mathrm{CM}$, congestive heart failure, old age, hypertension, route of administration of $\mathrm{CM}$, and the use of other nephrotoxic drugs. ${ }^{13,20}$

Any condition associated with decreased effective circulating volume enhances vulnerability with regard to CIN. ${ }^{19}$ Of course, other causes of acute renal failure, such as atheromatous embolic disease, ischaemia, prerenal azotemia, sepsis, or other nephrotoxins should always be considered, particularly if CIN is suspected in a patient without known risk factors. For example, CIN might be mistaken for cholesterol crystal embolisation after intravascular catheterisation.

When comparing the renal effects of different vascular contrast agents in patients with normal renal function, there are inconclusive results in the literature. Patients with no pre-existing renal impairments have been shown to be highly resistant to CIN, even when using ionic highosmolar CM. ${ }^{3,21}$ Unfortunately, though, patients with pre-existing renal impairments are to a large extent also the patients requiring angiography ${ }^{22}$ and in this subpopulation high doses of $\mathrm{CM}$ increases the risk for $\mathrm{CIN}^{23}$

\section{Substance groups}

For over 50 years the various available $\mathrm{CM}$ have been based on triiodobenzene. Their characteristics vary due to the osmolality and ionicity of the product. Earlier, during the time of high-osmolar CM (which have osmolalities approximately 6 times higher than plasma found in broad clinical use), it made sense to differentiate $\mathrm{CM}$ with regard to osmolality. However, already then it was obvious that many of the side-effects were actually caused by the electric charge. The current use of lowosmolar CM (which still have considerably higher osmolality than plasma) and isoosmolar CM is widespread and it may be that the subdivision of $\mathrm{CM}$ according to their osmolality should be reconsidered. This is so since iso-osmolar CM are dimers, thus revealing greater viscosities than the monomeric low-osmolar CM (Table I). As outlined below, this difference can be of importance for renal and systemic haemodynamics.

\section{Clinical studies}

Use of the more modern CM that are low- or iso-osmolar has reduced the likelihood of CIN compared with high-osmolar CM.

In a prospective, randomised study involving 1196 patients undergoing angiocardiography, Rudnick et al. ${ }^{5}$ found no differences in incidence of nephropathy between patients receiving iohexol (lowosmolar, $780 \mathrm{mOsm} / \mathrm{kg} \mathrm{H}_{2} \mathrm{O}$ ) and patients receiving diatrizoate (high-osmolar, 1870 $\mathrm{mOsm} / \mathrm{kg} \mathrm{H}_{2} \mathrm{O}$ ) among low-risk patients (patients without diabetes who had a baseline serum creatinine concentration of less than $1.5 \mathrm{mg} / \mathrm{dl}(133 \mu \mathrm{mol} / \mathrm{l}))$. However, in patients without diabetes whose serum creatinine concentrations were higher than 1.5 $\mathrm{mg} / \mathrm{dl}$, the incidence of nephropathy was reduced from 27.0 to $12.2 \%$ with the use of iohexol. ${ }^{5}$ For patients with diabetes, the incidence was reduced from 47.7 to $33.3 \%$. Overall, patients receiving high-osmolar CM were 3.3 times as likely to develop CIN as those receiving low-osmolar CM. For all practical purposes, all the newer lowosmolar or iso-osmolar agents are considered to be the agents of choice in patients at higher than usual risk for the development of CIN. ${ }^{19}$

Some comparative studies in patients with pre-existing renal impairment have shown similar susceptibility for CIN with both nonionic monomeric and nonionic dimeric $\mathrm{CM}^{24-26}$ whereas other trials have concluded that iso-osmolar CM have advantages with regard to the occurrence of CIN in renally impaired patients. ${ }^{1,27}$ In particular Aspelin et al. ${ }^{1}$ in the NEPHRIC study concluded that iohexol $(N=65)$ was significantly more nephrotoxic than the nonionic dimer iodixanol $(N=64)$ in patients with pre-existing chronic renal failure undergoing coronary or aortofemoral angiography. These results have received considerable attention due to the conclusions of the authors, but also due to some serious shortcomings of the study design. Previous studies have shown that critical factors for the susceptibility of CIN are duration of diabetes, metabolic status and renal function before $\mathrm{CM}$ injection. ${ }^{8}$ The investigated groups in the study of Aspelin and co-workers ${ }^{1}$ were significantly different in all of these parameters and thus the outcome of the study is more likely to reflect the differences of the studied populations rather than differences of the administered CM with regard to CIN. In addition, the hydration regimen was not pursued with vigour, as indicated by considerable variance in fluid intake.

The conclusion of the NEPHRIC study that the use of iso-osmolar CM, as opposed to low-osmolar $\mathrm{CM}$, results in reduced incidence of CIN is not in line with our current understanding of CIN, as mentioned below, and contradicts studies in which the use of iso-osmolar CM conferred no advantage. ${ }^{28,29}$ In light of the controversy whether patients at risk actually benefit from iso-osmolar $\mathrm{CM}^{1,27}$ or not ${ }^{24-26}$ and the experimental data on physiologic/pathophysiologic renal mechanisms that do not support any beneficial effects of iso-osmolar $\mathrm{CM}$, the $\mathrm{CM}$ of choice still remains an open question..$^{30}$

\section{Mechanisms of CIN}

There is a particularly vulnerable kidney region in the deeper portion of the outer medulla. This is an area remote from the vasa recta that supply the renal medulla with blood. The reason for the vulnerability of the outer medullary portion of the nephron is the relative high oxygen requirements due to salt reabsorption. In this area of the kidney, the limbs of the loop of Henle exhibit hypoxic damage, for instance by perfusion with erythrocyte free solution. ${ }^{31}$ Oxygen delivery to the peripheral tissues can be impaired by $\mathrm{CM}$ due to an increase oxygen affinity of haemoglobin. ${ }^{32}$

It is not fully clear what the underlying mechanism is with regard to CIN. Several suggestions have been put forward and it is widely held that a combination of various mechanisms need to act in concert to cause CIN. ${ }^{19}$ Among these mechanisms, a reduction in renal perfusion caused by a direct effect of CM on the kidney and toxic effects on the tubular cells are generally recognised as important. However, the pathophysiological relevance of direct effects of $\mathrm{CM}$ on 
tubular cells remains disputed, ${ }^{19}$ as are the other proposed aetiologies.

By adding $\mathrm{CM}$ to the perfusate of the kidney, hypoxic injury to the region at risk is enhanced, probably by increasing renal vascular resistance. ${ }^{33}$ It has been shown that an iso-osmolar dimer with high viscosity (iodixanol) compromises blood flow to all regions of the kidney to a greater extent than low-osmolar, and even high-osmolar CM. ${ }^{29}$ However, it must be kept in mind that this decrease in perfusion was accompanied by profound systemic effects of iodixanol. Blood pressure in this study dropped considerably. ${ }^{29}$ In fact, it is well known that local renal hypoxia can be aggravated by the systemic effects of some $\mathrm{CM}$, such as transiently reduced cardiac output, ${ }^{34}$ and suboptimal pulmonary perfusion-ventilation relationship. ${ }^{35}$

Another study ${ }^{36}$ supports the particular potency of dimeric $\mathrm{CM}$ in causing renal hypoxia - the iso-osmolar CM iotrolan was also found to impair local $\mathrm{pO}_{2}$ to a greater extent than the low-osmolar CM iopromide.

The tubuloglomerular feedback (TGF) is a powerful mechanism in the control of renal vascular resistance and glomerular filtration and has often been taken as an explanation for the development of CIN Specifically, it is believed that increased osmotic pressure brought about by $\mathrm{CM}$ elicits renal ischaemia.

The macula densa cells of the thick ascending limb mediate the TGF by sensing $\mathrm{Na}^{+}, \mathrm{K}^{+}$, and $\mathrm{Cl}^{-}$concentrations in the tubular fluid via the $\mathrm{Na}^{+}-\mathrm{K}^{+}-2 \mathrm{Cl}^{-}$cotransporter. This transporter is effectively blocked by furosemide. The affinity for $\mathrm{Cl}^{-}$ is very low, so in a physiological setting there will always be enough $\mathrm{Na}^{+}$and $\mathrm{K}^{+}$to keep the system running; $\mathrm{Cl}^{-}$is the limiting factor. ${ }^{37,38}$ A widespread explanation for the development of CIN is that hyperosmotic $\mathrm{CM}$ causes an increased osmotic gradient at the macula densa, which activates the TGF and subsequently compromises renal blood flow and glomerular filtration. Obviously, this chain of events is not a likely explanation for CIN, and this has been shown already by pioneer experiments with retrograde perfusions of the tubule. In this setting, osmolality has no effect on the TGF. $^{37,38}$ The ruling out of the osmotic diuresis theory is further supported by experiments using mannitol, an osmotic diuretic. Increases in osmolality, such as after mannitol infusion or after CM application, decrease $\mathrm{NaCl}$ concentration at the macula densa, however, simultaneously increasing tubular flow. Therefore, the resulting net change in the amount of $\mathrm{NaCl}$ passing the macula densa is negligible. ${ }^{39}$ Moreover, furosemide, a known blocker of the TGF, does not decrease serum creatinine after application of CM, which is usually the parameter taken to indicate CIN. ${ }^{2}$ Blocking the transport using furosemide should dramatically lower local oxygen consumption and alleviate the reduced oxygen supply. In fact, this has been demonstrated to occur in experiments in rats showing that outer medullary $\mathrm{pO}_{2}$ is elevated after furosemide. ${ }^{40}$ However, furosemide given just before angiography fails to limit increases in serum creatinine after $\mathrm{CM}$ application, indicating that yet other mechanisms are involved in CIN. ${ }^{2}$

Among the often-discussed mechanisms, endothelin and adenosine have received much attention. The effects of endothelin on vascular beds is very dependent upon the receptor subtype activation, but both subtypes of receptors (ET-A and ET-B) are thought to mediate the vascular actions of endothelins in human blood vessels. ${ }^{41}$

Endothelin has been suggested as a key element in the scenario leading to CIN because of the elevated endothelin levels in plasma and urine that are found after CM application. ${ }^{42-44}$ Further support for the role of endothelin in CIN is derived from the observed enhancement of endothelin transcription and release from endothelial cells in response to $\mathrm{CM}^{45}$ Finally, in patients with impaired renal function, the increase in endothelin after giving CM is exaggerated. ${ }^{46}$ A potential beneficial effect of endothelin in preventing CIN may be mediated by the ET-B mediated effects, such as vasorelaxation. Thus, a selective ETA receptor blockade could prove to be effective in the prevention of CIN. Indeed, a positive effect of ET-A selective blockade on the renal outer medullary hypoxic response to $\mathrm{CM}$ has been reported in the normal rat. ${ }^{47}$ However, when both ET-A and ET-B receptors are blocked in humans receiving $\mathrm{CM}$, serum creatinine concentration rises to a greater extent than in patients receiving placebo and the CIN incidence is significantly increased in the patients who received combined ET-A and ET-B blockade. $^{48}$

Diabetes mellitus is among the most important risk factors for CIN and diabetics often have endothelial dysfunction of renal vessels. In this setting, NO is suppressed in the renal microvasculature which contributes to the endothelial dysfunction. ${ }^{49}$ Superoxide is a scavenger of NO and may cause the attenuated NO activity found in the diabetic renal microvasculature. Indeed, superoxide production has been found to be increased in renal cortical tissue from diabetic rats ${ }^{50}$ and the afferent and efferent arteriolar vasoconstrictor response to NOS inhibition is impaired. ${ }^{51}$ Taken together, superoxide, and perhaps other reactive oxygen species (ROS) may be crucial in the development of CIN. Since ROS are extracellular signalling molecules, they may be significant in mediating the part of the endothelin effects. ${ }^{57}$

Due to the possible role of ROS in CIN, clinical trials have been undertaken to prevent $\mathrm{CIN}$ by scavenging ROS. ${ }^{52-55}$ In these trials, $\mathrm{N}$-acetylcysteine was given and showed a positive outcome in four studies. ${ }^{53-56}$ However, this recommendation is by no means unequivocal. ${ }^{57}$

In analogy to endothelin, a prominent role in causing CIN has also been suggested for adenosine. The renal vasculature of diabetics reveals an enhanced sensitivity to adenosine, thus it has been suggested that adenosine is a particularly important contributor to CIN in patients with this metabolic disorder. ${ }^{58}$ However, the role for adenosine in CIN may be considerably overestimated. For instance, in normal rats, ${ }^{59} \mathrm{~A}_{1}$-receptor blockade fails to alleviate medullary hypoperfusion and hypoxia in response to CM. Moreover, the general reduction in renal plasma flow and GFR caused by $\mathrm{CM}$ is not attributable to enhanced adenosine action. ${ }^{60}$

$\mathrm{CM}$ can also have direct cytotoxic effects on renal tubular cells. A perturbation of mitochondrial enzyme activity and mitochondrial membrane potential is found under ex vivo conditions in a proximal tubule cell line. ${ }^{61}$ Noteably, low-osmo- 
lar monomeric CM cause less damage than iso-osmolar dimeric CM and ionic compounds reveal the most profound effect. ${ }^{61}$ In the more distal segments of the kidney, $\mathrm{CM}$ can cause apoptosis, as indicated in another cell line model. ${ }^{62}$ Apoptosis may be brought about by hypoxic damage ${ }^{63}$ and by a direct influence on these cells. ${ }^{62}$

A quite simple mechanism that seems to be of paramount importance for the development of CIN has hitherto attracted rather little attention: the rhelogical properties of CM. The viscosity of the fluid is of particular importance with regard to the renal vascular bed, since the length of the capillaries that supply the renal medulla with blood are extremely long. Although the vasa recta have the same diameter as usual capillaries, they are several $\mathrm{cm}$ long. This increases vascular resistance, as indicated by Poiseuille's law: (Equation 1$) \mathrm{R}=\eta$ ${ }^{\star} 8^{\star} 1 / \Pi^{\star}{ }^{\star}{ }^{4}(\eta$ is viscosity, 1 refers to the length of the vessel and $r$ is the radius).

The viscosity of the blood flowing through the vasa recta is maintained very low in order to minimise the large resistance caused by capillary length. This is brought about by the Fåhraeus-Lindqvist effect and plasma skimming. The Fåhraeus-Lindqvist effect guarantees that blood viscosity in the capillary is very low. In fact, it is not much higher than plasma viscosity. This is so due to the high-flow velocity of the erythrocytes flowing through capillaries (single line flow). In effect haematocrit is very low in these vessels.

The low haematocrit is further brought about by plasma skimming: The afferent arterioles separate from the interlobular arteries at a right angle. Since the erythrocytes are concentrated in the centre of the interlobular arteries (laminar flow), the plasma-rich blood near the endothelium is skimmed off into the juxtamedullary afferent arterioles.

Taking these general considerations in to account, it is clear that iso-osmolar CM are not a priori superior to low-osmolar agents, since the dimeric iso-osmolar CM have very high viscosities. Therefore, isoosmolar CM should impair renal medullary blood flow to a greater extent than low-osmolar agents, which indeed seems to be the case, as indicated by the particularly reduced $\mathrm{pO}_{2}$ levels caused by iso-osmolar CM (Fig. 1).

Augmented fluid viscosity caused by dimeric iso-osmolar CM may be of even more importance in the renal tubule. Under normal conditions, tubular fluid is of lower viscosity than plasma, as the ultrafiltrate contains very few plasma proteins. Use of dimeric iso-osmolar CM will increase tubular fluid viscosity dramatically and thereby increase the resistance to flow in renal tubules. ${ }^{64}$ In consequence, renal interstitial pressure may take on values as high as $50 \mathrm{mmHg}$ (Fig. 2). Such pressure will dramatically decrease renal medullary flow and decrease GFR. Volume expanding the patient before application of $\mathrm{CM}$ will markedly alleviate this effect since the CM will not become as concentrated in the renal collecting ducts. This may be the mechanism responsible for the generally accepted best way to prevent CIN: periprocedural hydration. ${ }^{2,65,66}$

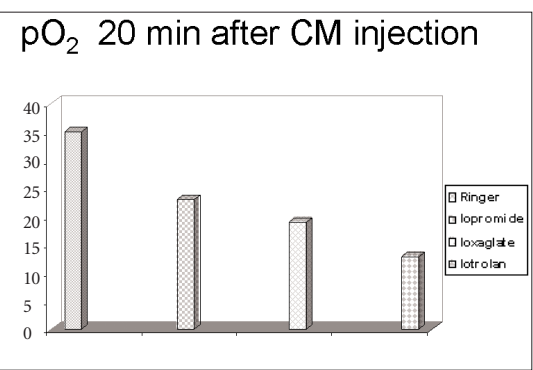

Fig. 1. Medullary hypoxia induced by CM compared with Ringer's solution. Reduction in $\mathrm{pO}_{2}$ is greatest for iotrolan (iso-osmolal non-ionic dimer) followed by ioxaglate (low-osmolal ionic dimer). lopromide (low-osmolal monomer) had the least effect of the CM. Data from Liss et al. ${ }^{36}$

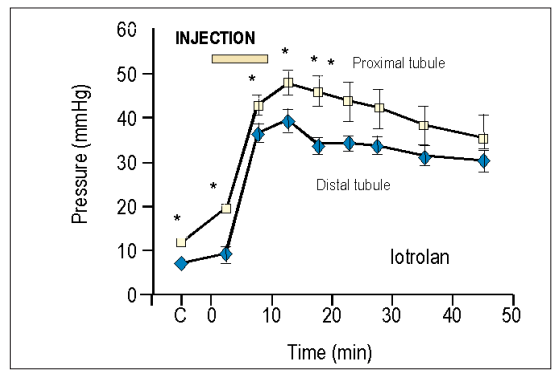

Fig. 2. Renal tubular pressures after injection of an iso-osmolar non-ionic CM, iotrolan. (Data from Ueda et al. Acta Radiol 1998; 39: 90 - 95).

\section{Conclusions}

The current understanding of CIN development now includes the rheological properties of a fluid. Resistance depends on fluid viscosity, not osmolality (Poiseuille's law). Thus, perhaps too much attention has been directed to the osmolality of different $\mathrm{CM}$, while neglecting the impact of other physicochemical properties. Well-controlled animal studies cannot confirm that iso-osmolar CM are superior with regard to the occurrence of CIN. The contrary seems to be the case.

\section{References}

1. Aspelin P, Aubry P, Fransson SG, et al. Nephrotoxic effects in high-risk patients undergoing angiography. N Engl J Med 2003; 348: 491 - 499.

2. Solomon R, Werner C, Mann D, et al. Effects of saline, mannitol, and furosemide to prevent acute decreases in renal function induced by radiocontrast agents. N Engl J Med 1994; 331: 1416-1420.

3. Barrett BJ, Parfrey PS, Vavasour HM, et al. Contrast nephropathy in patients with impaired renal function: high versus low osmolar media. Kidney Int 1992; 41: 1274-1279.

4. Manske CL, Sprafka JM, Strony JT, et al. Contrast nephropathy in azotemic diabetic patients undergoing coronary angiography. Am J Med 1990; 89: 615-620.

5. Rudnick MR, Goldfarb S, Wexler L, et al. Nephrotoxicity of ionic and nonionic contrast media in 1196 patients: a randomized trial. The Iohexol Cooperative Study. Kidney Int 1995; 47: 254-261.

6. Taliercio CP, Vlietstra RE, Ilstrup DM, et al. A randomized comparison of the nephrotoxicity of iopamidol and diatrizoate in high-risk patients undergoing cardiac angiography. J Am Coll Cardio 1991; 17: 384-390.

7. Levy EM, Viscoli CM, Horwitz RI. The effect of acute renal failure on mortality. A cohort analysis. JAMA 1996; 275: 1489-1494.

8. Waybill MM, Waybill PN. Contrast media-induced nephrotoxicity: identification of patients at risk and algorithms for prevention. J Vasc Interv Radiol 2001; 12: 3-9.

9. Hou SH, Bushinsky DA, Wish JB et al. Hospitalacquired renal insufficiency: a prospective study. Am J Med 1983; 74: 243-248.

10. Rihal CS, Textor SC, Grill DE, et al. Incidence and prognostic importance of acute renal failure after percutaneous coronary intervention. Circulation 2002; 105: 2259-2264.

11. Berg KJ. Nephrotoxicity related to contrast media. Scand J Urol Nephrol 2000; 34: 317-322.

12. Nikolsky E, Aymong ED, Dangas G, et al. Radiocontrast nephropathy: identifying the highrisk patient and the implications of exacerbating renal function. Rev Cardiovasc Med 2003; 4: Suppl 1:S7-S14.

13. Berns AS. Nephrotoxicity of contrast media. Kidney Int 1989; 36: 730-740.

14. McCullough PA, Wolyn R, Rocher LL, et al. Acute renal failure after coronary intervention: incidence, risk factors, and relationship to mortality. Am J Med 1997; 103: 368-375.

15. Parfrey PS, Griffiths SM, Barrett BJ, et al. Contrast material-induced renal failure in patients with diabetes mellitus, renal insufficiency, or both. A prospective controlled study. N Engl J Med 1989; 
19: 143-149.

16. Rich MW, Crecelius CA. Incidence, risk factors, and clinical course of acute renal insufficiency after cardiac catheterization in patients 70 years of age or older. A prospective study. Arch Intern Med 1990; 150: 1237-1242.

17. Thomsen HS, Morcos SK. Radiographic contrast media. BJU Int 2000; 86: Suppl 1, 1-10.

18. Gruberg L, Pinnow E, Flood R, et al. Incidence, management, and outcome of coronary artery perforation during percutaneous coronary intervention. Am J Cardiol 2000; 86: 680-682.

19. Thomsen HS, Morcos SK. Contrast media and the kidney. European Society of Urogenital Radiology (ESUR) guidelines. Br J Radiol 2003; 76: 513-518.

20. Deray G, Cacoub P, Jacquiaud C, et al. Renal tolerance for ioxaglate in patients with chronic renal failure. Radiology 1991; 179: 395-397.

21. Schwab SJ, Hlatky MA, Pieper KS, et al. Contrast nephrotoxicity: a randomized controlled trial of a nonionic and an ionic radiographic contrast agent. N Engl J Med 1989; 19: 149-153.

22. Solomon R. Contrast-medium-induced acute renal failure. Kidney Int 1998; 53: 230-242.

23. Cigarroa RG, Lange RA, Williams RH, et al. Dosing of contrast material to prevent contrast nephropathy in patients with renal disease. Am J Med 1989; 86: 649-652

24. Andrew E, Berg KJ, Nossen JO, et al. Renal effects of iodixanol in patients: a comparison with other radiographic contrast media. Acad Radiol 1996; 3: Suppl 2: S440-S443.

25. Carraro M, Malalan F, Antonione R, et al. Effects of a dimeric vs a monomeric nonionic contrast medium on renal function in patients with mild to moderate renal insufficiency: a double blind, randomized clinical trial. Eur Radiol 1998; 8: 144-147.

26. Jakobsen JA. Renal experience with Visipaque. Eur Radiol 1996; 6: Suppl 2: S16-S19.

27. Chalmers N, Jackson RW. Comparison of iodixanol and iohexol in renal impairment. Br J Radiol 1999; 72: 701-703.

28. Deray G, Bagnis C, Jacquiaud C, et al. Renal effects of low and isoosmolar contrast media on renal hemodynamic in a normal and ischemic dog kidney. Invest Radiol 1999; 34: 1-4.

29. Lancelot E, Idee JM, Couturier V, et al. Influence of the viscosity of iodixanol on medullary and cortical blood flow in the rat kidney: a potential cause of nephrotoxicity. J Appl Toxicol 1999; 19: 341-346.

30. Sandler CM. Contrast-agent-induced acute renal dysfunction-is iodixanol the answer? $N$ Engl J Med 2003; 348: 551-553.

31. Brezis M, Rosen S, Silva P, et al. Selective vulnerability of the medullary thick ascending limb to anoxia in the isolated perfused rat kidney. J Clin Invest 1984; 73: 182-190.

32. Kim SJ, Salem MR, Joseph NJ, et al. Contrast media adversely affect oxyhemoglobin dissociation. Anesth Analg 1990; 71: 73-76.

33. Heyman SN, Brezis M, Reubinoff CA, et al. Acute renal failure with selective medullary injury in the rat. J Clin Invest 1988; 82: 401-412.
34. Dawson P. Cardiovascular effects of contrast agents. Am J Cardiol 1989; 64: 2E-9E.

35. Neagley SR, Vought MB, Weidner WA, et al. Transient oxygen desaturation following radiographic contrast medium administration. Arch Intern Med 1986; 146: 1094-1097.

36. Liss P, Nygren A, Erikson U, et al. Injection of low and iso-osmolar contrast medium decreases oxygen tension in the renal medulla. Kidney Int 1998; 53: 698-702

37. Briggs JP, Schnermann J, Wright FS. Failure of tubule fluid osmolarity to affect feedback regulation of glomerular filtration. Am J Physiol 1980; 239: F427-F432.

38. Schnermann J, Ploth DW, Hermle M. Activation of tubulo-glomerular feedback by chloride transport. Pflugers Arch 1976; 362: 229-240.

39. Leyssac PP, Holstein-Rathlou NH, Skott O. Renal blood flow, early distal sodium, and plasma renin concentrations during osmotic diuresis. Am J Physiol Regul Integr Comp Physiol 2000; 279: R1268-R1276.

40. Liss P, Nygren A, Ulfendahl HR, et al. Effect of furosemide or mannitol before injection of a nonionic contrast medium on intrarenal oxygen tension. Adv Exp Med Biol 1999; 471: 353-359.

41. Seo B, Oemar BS, Siebenmann R, et al. Both ETA and ETB receptors mediate contraction to endothelin-1 in human blood vessels. Circulation 1994; 89: 1203-1208.

42. Bagnis C, Idee JM, Dubois M, et al. Role of endothelium-derived nitric oxide-endothelin balance in contrast medium-induced acute renal vasoconstriction in dogs. Acad Radiol 1997; 4: 343348 .

43. Clark BA, Kim D, Epstein FH. Endothelin and atrial natriuretic peptide levels following radiocontrast exposure in humans. Am J Kidney Dis 1997; 30: $82-86$

44. Heyman SN, Clark BA, Kaiser N, et al. Radiocontrast agents induce endothelin release in vivo and in vitro. J Am Soc Nephrol 1992 3:58-65.

45. Oldroyd SD, Morcos SK. Endothelin: what does the radiologist need to know? Br I Radiol 2000; 73: 1246-1251.

46. Fujisaki K, Kubo M, Masuda K, et al. Infusion of radiocontrast agents induces exaggerated release of urinary endothelin in patients with impaired renal function. Clin Exp Nephrol 2003; 7: 279-283.

47. Liss P, Carlsson PO, Nygren A, et al. Et-A receptor antagonist BQ123 prevents radiocontrast mediainduced renal medullary hypoxia. Acta Radiol 2003; 44: 111-117.

48. Wang A, Holcslaw T, Bashore TM, et al. Exacerbation of radiocontrast nephrotoxicity by endothelin receptor antagonism. Kidney Int 2000; 57: $1675-1680$

49. Schnackenberg CG. Physiological and pathophysiological roles of oxygen radicals in the renal microvasculature. Am J Physiol Regul Integr Comp Physiol 2002; 282: R335-R342.

50. Ishii N, Patel KP, Lane PH, et al. Nitric oxide synthesis and oxidative stress in the renal cortex of rats with diabetes mellitus. J Am Soc Nephrol 2001; 12: $1630-1639$
51. Palm F, Cederberg J, Hansell P, et al. Reactive oxygen species cause diabetes-induced decrease in renal oxygen tension. Diabetologia 2003; 46: 11531160.

52. Briguori C, Manganelli F, Scarpato $\mathrm{P}$, et al. Acetylcysteine and contrast agent-associated nephrotoxicity. J Am Coll Cardiol 2002; 40: 298303.

53. Diaz-Sandoval LJ, Kosowsky BD, Losordo DW Acetylcysteine to prevent angiography-related renal tissue injury (the APART trial). Am J Cardiol 2002; 89: 356-358

54. Shyu KG, Cheng JJ, Kuan P. Acetylcysteine protects against acute renal damage in patients with abnormal renal function undergoing a coronary procedure. J Am Coll Cardiol 2002; 40: 1383-1388.

55. Tepel M, van der Giet M, Schwarzfeld C, et al Prevention of radiographic-contrast-agentinduced reductions in renal function by acetylcysteine. N Engl J Med 2000; 20: 180-184.

56. Kay J, Chow WH, Chan TM, et al. Acetylcysteine for prevention of acute deterioration of renal function following elective coronary angiography and intervention: a randomized controlled trial. JAMA 2003; 289: 553-558.

57. Durham JD, Caputo C, Dokko J, et al. A randomized controlled trial of $\mathrm{N}$-acetylcysteine to prevent contrast nephropathy in cardiac angiography. Kidney Int 2002; 62: 2202-2207.

58. Pflueger A, Larson TS, Nath KA, et al. Role of adenosine in contrast media-induced acute renal failure in diabetes mellitus. Mayo Clin Proc 2000; 75: 1275-1283.

59. Liss P, Carlsson PO, Palm F, et al. Adenosine A (1) receptors in contrast media-induced renal dysfunction in the normal rat. Eur Radiol 2004; 14: 1297-1302.

60. Oldroyd SD, Fang L, Haylor JL, et al. Effects of adenosine receptor antagonists on the responses to contrast media in the isolated rat kidney. Clin $\mathrm{Sci}$ (Lond) 2000; 98: 303-311

61. Hardiek K, Katholi RE, Ramkumar V, et al. Proximal tubule cell response to radiographic contrast media. Am J Physiol Renal Physiol 2001; 280: F61-F70.

62. Hizoh I, Strater J, Schick CS, et al. Radiocontrastinduced DNA fragmentation of renal tubular cells in vitro: role of hypertonicity. Nephrol Dial Transplant 1998; 13: 911-918.

63. Beeri R, Symon Z, Brezis M, et al. Rapid DNA fragmentation from hypoxia along the thick ascending limb of rat kidneys. Kidney Int 1995; 47: 1806-1810.

64. Ueda J, Nygren A, Hansell P, et al. Influence of contrast media on single nephron glomerular filtration rate in rat kidney. A comparison between diatrizoate, iohexol, ioxaglate, and iotrolan. Acto Radiol 1992; 33: 596-599.

65. Murphy SW, Barrett BJ, Parfrey PS. Contras nephropathy. J Am Soc Nephrol 2000; 11: 177-182.

66. Trivedi HS, Moore H, Nasr S, et al. A randomized prospective trial to assess the role of saline hydration on the development of contrast nephrotoxicity. Nephron Clin Pract 2003; 93: C29-C34. 